\title{
Caracterização de nanofilmes de celulose nanofibrilada obtida em diferentes consistências
}

\author{
Characterization of fibrillated cellulose \\ nanofilms obtained at different consistencies
}

\author{
Daniele Cristina Potulski ${ }^{1}$, Lívia Cássia Viana², Graciela Ines Bolzon de Muniz ${ }^{3}$, \\ Alan Sulato de Andrade ${ }^{4}$ e Umberto Klock ${ }^{5}$
}

\begin{abstract}
Resumo
A celulose nanofibrilada destaca-se por apresentar propriedades físicas e mecânicas singulares, como alta densidade, menor capacidade de absorção de água e elevada resistência a tração e arrebentamento. $O$ objetivo principal deste trabalho foi produzir e avaliar filmes de celulose nanofibrilada obtida pelo processo de desfibrilação mecânica a partir de diferentes consistências. Polpa Kraft de Pinus sp. não branqueada, preparada em diferentes consistências: $0,5 \%, 1,0 \%$ e 1,5\% e processadas mecanicamente no moinho desfibrilador Super Masscolloider Masuko Sangyo utilizando 20 passes foi utilizada na obtenção da celulose nanofibrilada. Para visualização das estruturas e dimensões da celulose nanofibrilada foi aplicada a técnica de microscopia eletrônica de transmissão e força atômica. O índice de cristalinidade da celulose nos filmes foi obtido aplicando a técnica de difração de Raios- $X$. Os filmes previamente acondicionados (T: $23 \pm 2{ }^{\circ} \mathrm{C}$ e UR: de $50 \pm 2 \%$ ) foram destinados à realização dos ensaios físicos (gramatura, densidade aparente e absorção). Os resultados mostram que os valores dos índices de cristalinidade foram de 69,8 ; 72,1 e $72,2 \%$ para a celulose nanofibrilada obtidas a partir das consistências de 1,0; 0,5 e 1,5\%, respectivamente. Os valores de gramatura foram de 56,92 a $63,08 \mathrm{~g} / \mathrm{m}^{2}$ e estão dentro da variação proposta nesse trabalho. A densidade aparente foi igual a 1,22 (C0,5\%); 1,35 (C1,0\%) e 1,10 g/ $\mathrm{cm}^{3}(\mathrm{C} 1,5 \%)$. O ensaio de absorção de água pelo método Cobb não mostrou diferença estatisticamente significativa entre os tratamentos avaliados. A presença de nanofibrilas aumenta a densidade aparente e diminui a absorção de água em filmes nanoestruturados. As diferentes consistências estudadas não influenciaram nas características e propriedades físicas dos filmes.
\end{abstract}

Palavras-chave: nanocelulose, filmes, Pinus, densidade, absorção Cobb.

\begin{abstract}
Nanofibrillated cellulose stands out for having remarkable physical and mechanical properties such as high density, lower capacity of water absorption and high tensile and burst resistance. The main objective of this research was to produce and evaluate nanostructured cellulosic films obtained by mechanical defibrillation process from three different consistencies: $0.5 \%, 1.0 \%$ and $1.5 \%$. Undelignified Kraft pulp Pinus sp. prepared in different consistencies were used: $0.5 \%, 1.0 \%$ and $1.5 \%$ and processed by mechanical defibrillation in a grinder Super Masscolloider Masuko Sangyo by 20 passes. Transmission Electron Microscopy and Atomic Force was used to visualize the structures and dimensions of the cellulose nanofibers. The cellulosic crystallinity index on the films was obtained by using X-Ray diffraction. The previously conditioned films (T: $23 \pm 2{ }^{\circ} \mathrm{C}$ e UR: de $50 \pm 2 \%$ ) were destined to achieve physical tests (thickness, grammage, apparent density and absorption). The results show that the values of the crystallinity index was 69.8; 72.1 and $72.2 \%$ for nanofibrillated cellulose to $0.5,1.0$ and $1.5 \mathrm{wt} \%$, respectively. The grammage values were from 56.92 to $63.08 \mathrm{~g} / \mathrm{m}^{2}$, and are within the range proposed in this work. The apparent density was $1.22(\mathrm{C} 0.5 \%), 1.35(\mathrm{C} 1.0 \%)$ and $\left.1.10 \mathrm{~g} / \mathrm{cm}^{3} \mathrm{C} 1.5 \%\right)$. The Cobb Test showed no statistical significant difference among the treatments. The presence of nanofibrils increases density and decreases the water absorption of nanostructured films. The different consistencies did not influence the characteristics and physical properties of the films.
\end{abstract}

Keywords: nanocellulose, films, Pine, density, Cobb absorption

${ }^{1}$ Doutoranda no Departamento de Engenharia e Tecnologia Florestal. UFPR-Universidade Federal do Paraná. Av: Prefeito Lothário Meissner, 632 - Jardim Botânico - 80210-170 - Curitiba, PR. E-mail: danielepotulski@ufpr.br.

${ }^{2}$ Doutora em Engenharia Florestal. UFPR-Universidade Federal do Paraná. Av: Prefeito Lothário Meissner, 632 - Jardim Botânico - 80210-170 - Curitiba, PR. E-mail: licvianna@gmail.com.

3Professora titular do Departamento de de Engenharia e Tecnologia Florestal. UFPR-Universidade Federal do Paraná. Av: Prefeito Lothário Meissner, 632 - Jardim Botânico - 80210-170 - Curitiba, PR. E-mail: gbmunize@ufpr.br.

${ }^{4}$ Professor Adjunto do Departamento de de Engenharia e Tecnologia Florestal. UFPR-Universidade Federal do Paraná. Av: Prefeito Lothário Meissner, 632 - Jardim Botânico - 80210-170 - Curitiba, PR. E-mail: alansulato@gmail.com.

${ }^{5}$ Professor Associado do do Departamento de de Engenharia e Tecnologia Florestal. UFPR-Universidade Federal do Paraná. Av: Prefeito Lothário Meissner, 632 - Jardim Botânico - 80210-170 - Curitiba, PR. E-mail: klockuer@ufpr.br.

Sci. For., Piracicaba, v. 44, n. 110, p. 361-372, jun. 2016 DOI: dx.doi.org/10.18671/scifor.v44n110.09 


\section{INTRODUÇÃO}

As crescentes buscas por novas tecnologias que visem à utilização de matérias primas renováveis, biodegradáveis, de menor impacto ambiental e que ao mesmo tempo apresentem propriedades diferenciadas, tem estimulado o aumento de pesquisas com a celulose no campo da nanotecnologia.

A celulose nanofibrilada consiste em um material obtido pela desintegração da celulose submetida a um processo mecânico de homogeneização, no qual é degradada, promovendo a exposição e abertura das superfícies anteriormente situadas no interior das fibras, as fibrilas e as microfibrilas (TURBAK et al., 1983 citados por ANDRESEN et al., 2006; GARDNER et al., 2008; KOLAKOVIC et al., 2011). Este processo ocasiona um aumento da superfície externa, possibilitando uma maior área de contato e maior capacidade de gerar fortes ligações de hidrogênio entre as microfibrilas da parede celular, produzindo um material com alta resistência.

Um dos produtos obtidos a partir da celulose por meio de processos mecânicos, químicos, físicos e/ou biológicos (FRONE et al., 2011), pode ser chamada de celulose nanofibrilada (CNF) ou nanofibrillated cellulose (NFC) do inglês. Outros termos genéricos como, celulose microfibrilada (CMF), nanofibras de celulose e nanofibrilas de celulose, por exemplo, têm sido usados para descrever nanoestruturas de celulose com pelo menos uma de suas dimensões em escala nanométrica, com diâmetros inferiores a $100 \mathrm{~nm}$ e comprimentos de vários micrômetros (STELTE; SANADI, 2009; SEHAQUI et al., 2011; SYVERUD et al., 2011).

Um dos métodos para obtenção da celulose nanofibrilada, sugerido em trabalhos recentes, trata-se da micro-moagem ou desfibrilação mecânica por utilizando um moinho (ABE; YANO 2010; PANTHAPULAKKAL; SAIN, 2012; WANG et al., 2013). Por meio desse processo, a celulose pode ser reduzida a diâmetros muito pequenos, atingindo de 25 a $100 \mathrm{~nm}$, sendo chamada de celulose microfibrilada, e diâmetros de 5 a $30 \mathrm{~nm}$ para a celulose nanofibrilada (SEHAQUI et al. 2011).

$\mathrm{O}$ "grinder" ou moinho consiste em um disco rotatório e um disco fixo com uma abertura ajustável entre eles para que por meio do contato mecânico as fibras de celulose sejam desfibriladas por forças de cisalhamento geradas pelas pedras de desfibrilação.

Além dos tratamentos mecânicos como homogeneização, moagem, refinação e cryocrushing, combinações de tratamentos químicos, mecânicos e enzimáticos já foram experimentados para a produção de nanocelulose. Dentre estes, pode-se citar a combinação de um sistema oxidativo utilizando o 2,2,6,6-2,2,6,6-Tetramethyl-1-piperidinyloxy (TEMPO) associado ao homogeinizador. Besbes et al. (2011) relata que o tempo de oxidação das fibras reduz acentuadamente a intensidade e o tempo do tratamento mecânico aplicado para a obtenção de CNF. A combinação de tratamentos químicos e mecânicos para obtenção de nanofibras têm sido relatados com sucesso em alguns trabalhos (IWAMOTO et al., 2008; OKAHISA et al., 2009).

Celulose nanofibrilada é um material ecologicamente correto e renovável, de baixo custo, disponível na natureza, com elevada área específica e excelentes propriedades mecânicas (HUBBE et al., 2008; NAKAGAITO et al., 2009). Seu uso tem encontrado um vasto campo de aplicações como reforços em compósitos, na composição de fármacos e cosméticos, produtos para construção, componentes de alimentos e embalagens, indústria eletrônica e elétrica, setor têxtil e biomedicina (IOELOVICH, 2008).

No setor florestal, a celulose nanofibrilada torna-se um material atraente para uso na indústria de celulose e papel, destacando-se por apresentar propriedades físicas e mecânicas singulares que juntamente com sua baixa densidade as tornam um material excelente para aplicações como revestimentos, na produção de filmes e papéis especiais, como aditivos na produção de papel (WANG; SAIN, 2007). Papéis produzidos com celulose nanofibrilada apresentam maior densidade, flexibilidade e melhores propriedades mecânicas. Podem ser opticamente transparentes, com baixo coeficiente de expansão térmica, baixa porosidade e apresentam excelentes propriedades de barreira ao oxigênio (NOGI; YANO, 2008; NOGI; YANO, 2009, GONZÁLEZ et al., 2012).

A abundância de aplicações e possíveis variações nas propriedades dos materiais em que a celulose nanofibrilada é aplicada justificam o interesse cada vez maior em estudos que proporcionem o conhecimento das principais propriedades e características deste tipo de nanocelulose.

Dentro desse contexto, este trabalho teve por objetivo produzir e avaliar filmes de celulose nanofibrilada obtida pelo processo de desfibrilação mecânica a partir de três diferentes consistências: $0,5 \%, 1,0 \%$ e $1,5 \%$. 


\section{MATERIAL E MÉTODOS}

Polpa celulósica Kraft industrial não branqueada (Kappa 44,5) de Pinus taeda foi utilizada para obtenção de celulose nanofibrilada.

A polpa, fornecida pela empresa no formato de cartão, passou pelo processo mecânico de desintegração das fibras no desintegrador tipo Bauer para promover a individualização das mesmas, seguindo posteriormente para as etapas de lavagem, centrifugação e determinação da umidade.

A polpa Kraft foi em seguida dispersa utilizando um desintegrador de polpa celulósica durante o tempo de cinco minutos para obtenção de uma suspensão de fibras homogeneizadas.

\section{Obtenção da celulose nanofibrilada}

Para obtenção da celulose nanofibrilada as amostras de polpa Kraft dispersas em água foram preparadas em diferentes consistências: 0,5\%, 1,0\% e 1,5\% (tratamentos C0,5\%; C1,0\% e C1,5\%) base massa seca, seguindo então para o processo mecânico de desfibrilação no moinho desfibrilador Super Masscolloider Masuko Sangyo (MKCA6-3; Masuko Sangyo Co., Ltd.) utilizando 20 passes e a freqüência constante de 1500rpm.

Após os 20 passes pelo moinho desfibrilador as suspensões nanocelulósicas obtidas nas diferentes consistências foram destinadas à produção de nanofilmes para a caracterização e determinação de suas propriedades físicas.

\section{Preparação dos filmes de celulose nanofibrilada}

Os filmes foram produzidos por meio da deposição da suspensão de celulose nanofibrilada sobre o conjunto papel filtro e tela de nylon para serem filtrados a vácuo. Estabeleceu-se a gramatura final desejada para os filmes de $60 \pm 5 \mathrm{~g} / \mathrm{m}^{2}$, sendo que para cada tratamento foram feitos três filmes. Após serem filtrados foram levados para secagem na máquina formadora de papel à temperatura de $90^{\circ} \mathrm{C}$ e pressão constante de $80 \mathrm{KPa}$. Os filmes foram então acondicionados em sala climatizada com temperatura de $23 \pm 2{ }^{\circ} \mathrm{C}$ e umidade relativa do ar de $50 \pm 2 \%$ para posterior determinação de suas propriedades.

\section{Caracterização Microscópica}

Para caracterização da celulose nanofibrilada pela técnica de microscopia eletrônica de transmissão (MET), foi utilizado um Microscópio Eletrônico de Transmissão JEOL JEM 1200EX-II, com resolução de 0,5 nm (600 mil X). O filme utilizado na análise foi preparado a partir da deposição de uma gota da solução de celulose nanofibrilada sobre o porta-amostra e em seguida seco à temperatura ambiente.

A celulose nanofibrilada foi caracterizada também pela técnica de microscopia de força atômica (MFA) por meio do equipamento Microscópio de Força Atômica Shimadzu SPM 9500, com resolução de $0,3 \mathrm{~nm}$ pelo modo de contato. O filme utilizado na análise foi preparado pela deposição de uma gota da solução de celulose nanofibrilada sobre uma lamínula de vidro que em seguida foi direcionado à estufa a $70 \pm 2{ }^{\circ} \mathrm{C}$ para secagem.

\section{Espectroscopia NIR}

A espectroscopia no infravermelho próximo (NIRS) foi usada como ferramenta para observar os principais picos presentes nos filmes, além de identificar possíveis mudanças nas intensidades dos picos que podem ser geradas pelo processo de desfibrilação a partir de diferentes consistências.

Os espectros na faixa do infravermelho próximo foram adquiridos no espectrômetro Bruker-Tensor 37, que utiliza a transformada de Fourier, acoplada ao programa OPUS. A aquisição espectral foi realizada no modo de absorbância na faixa de radiação de 10000 a $4000 \mathrm{~cm}^{-1} \mathrm{com}$ resolução espectral de $2 \mathrm{~cm}^{-1}$. Foram coletados dois espectros para cada filme.

\section{Índice de Cristalinidade}

Para determinação da cristalinidade dos nanofilmes produzidos foi utilizado um difratômetro de Raios-X da Shimadzu modelo XRD-7000 acoplado ao software XRD-6100/7000 v 5.0. A velocidade de varredura adotada foi de $1^{\circ} /$ min variando de 3 a $45^{\circ}$, utilizando radiação de $\mathrm{Cu}-\mathrm{K}$, comprimento de onda de 0,15418 $\mathrm{nm}$ e voltagem de $40 \mathrm{kV}$ com corrente de $20 \mathrm{~mA}$. 
O método adotado para determinação dos índices de cristalinidade foi o sugerido por Segal et al., (1959) (Equação 1), no qual o percentual de celulose cristalina é determinado pela diferença da intensidade entre o pico de maior intensidade (pico cristalino) e o pico de menor intensidade (porção amorfa). Para cada tratamento foram realizadas duas repetições, totalizando 6 valores de índice de cristalinidade.

$$
\% I C=\left(\frac{I-i}{I}\right) * 100
$$

Onde:

$\% \mathrm{IC}=$ Índice de cristalinidade

$\mathrm{I}=$ Pico de maior intensidade (pico cristalino);

$\mathrm{i}=$ Pico de menor intensidade (porção amorfa).

\section{Ensaios Físicos}

Para determinação dos ensaios físicos foi ensaiada uma amostra por filme, totalizando nove amostras. A Tabela 1 apresenta as normas utilizadas e as unidades, as quais, a propriedades são expressas.

Tabela 1. Propriedades físicas e normas.

Table 1. Physical properties and standard.

\begin{tabular}{lcc}
\hline Propriedades Físicas & Norma & Unidade \\
\hline Espessura & T411-om97 & $\mu \mathrm{m}$ \\
Gramatura & Norma T410-om02 & $\mathrm{g} / \mathrm{m}^{2}$ \\
Densidade Aparente & T220-om01 & $\mathrm{g} / \mathrm{cm}^{3}$ \\
Absorção pelo método Cobb60 & T441-om98* & $\mathrm{g} / \mathrm{m}^{2}$ \\
\hline
\end{tabular}

*Norma adaptada - tempo adotado 60s.

\section{Análise Estatística}

Os valores dos índices de cristalinidade, densidade aparente e Cobb, foram submetidos à análise de variância com médias comparadas pelo teste de Tukey a 5\% de probabilidade. O teste de Bartlett foi previamente realizado para testar a homogeneidade de variâncias que se mostraram homogêneas.

\section{RESULTADOS E DISCUSSÃO}

\section{Obtenção da celulose nanofibrilada}

A Figura 1 apresenta imagens da celulose nanofibrilada obtida a partir de diferentes consistências.

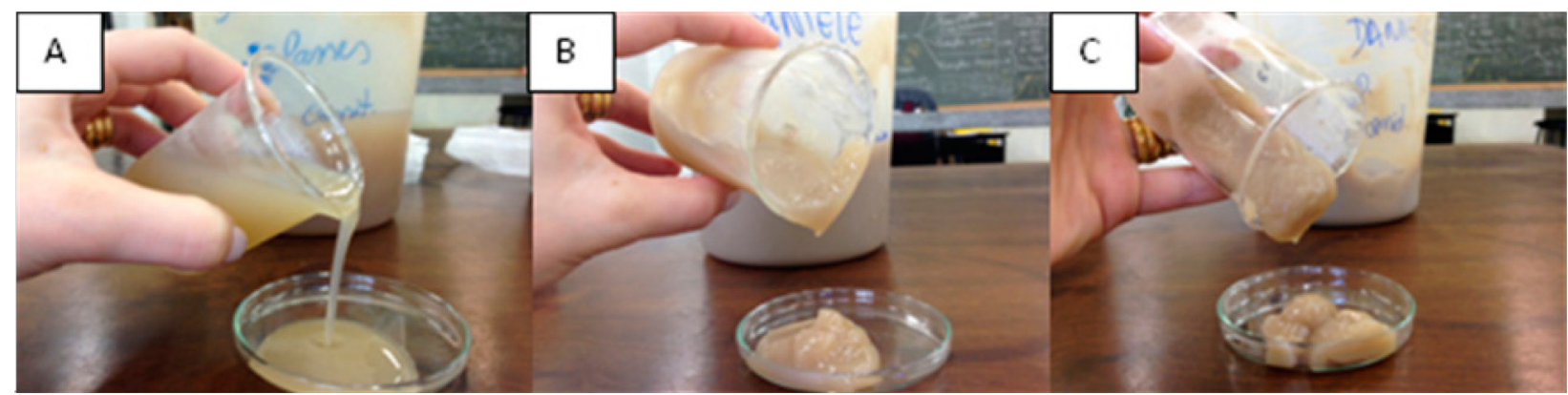

Figura 1. Celulose nanofibrilada (A): $0,5 \%$ de consistência (B): $1 \%$ de consistência $(C): 1,5 \%$ de consistência. Figure 1. Nanofibrillated cellulose (A): $0,5 \%$ wt (B): $1,0 \%$ wt (C): $1,5 \%$ wt.

Como pode ser observado na Figura 1 a celulose nanofibrilada obtida após 20 passes pelo moinho desfibrilador apresentou aspecto de gel para os três tratamentos considerados. Conforme também relatado por outros autores, métodos mecânicos de fibrilação da celulose para obtenção de celulose nanofibrilada a partir de fibras vegetais levam a formação de um gel com elevado teor de água, neste trabalho variando de 98,5 a 99,5\% (BESBES et al., 2011; KOLAKOVIC et al., 2011). A diferença verificada em relação à fluidez do gel deve-se justamente as diferentes porcentagens de celulose presentes em cada um dos tratamentos, sendo menor no tratamento 0,5\% (Figura 1A) e maior em 1,5\% (Figura 1C). Com o aumento da consistência ocorre uma redução na fluidez e consequente aumento na viscosidade. 
Géis de celulose nanofibrilada são composições estáveis caracterizados pelo baixo teor de sólidos, compostos por nanofibrilas e microfibrilas hidrofílicas com alta capacidade de desenvolver ligações de hidrogênio (LORANGER et al., 2012). A maior superfície específica da CNF, quando comprada as fibras de celulose, possibilita o aumento da exposição dos grupos hidroxilas disponíveis para interagir com a água, que por sua vez se encontra disponível elevada quantidade, ocorrendo a formação do gel.

\section{Caracterização Microscópica}

Na Figura 2A, B, C observam-se imagens obtidas por Microscopia Eletrônica de Transmissão, referentes à celulose nanofibrilada para as consistências de 0,5, 1,0 e 1,5\%, respectivamente. A Figura 2D apresenta as medidas dos diâmetros das nanofibrilas pertencentes ao tratamento C05\%.
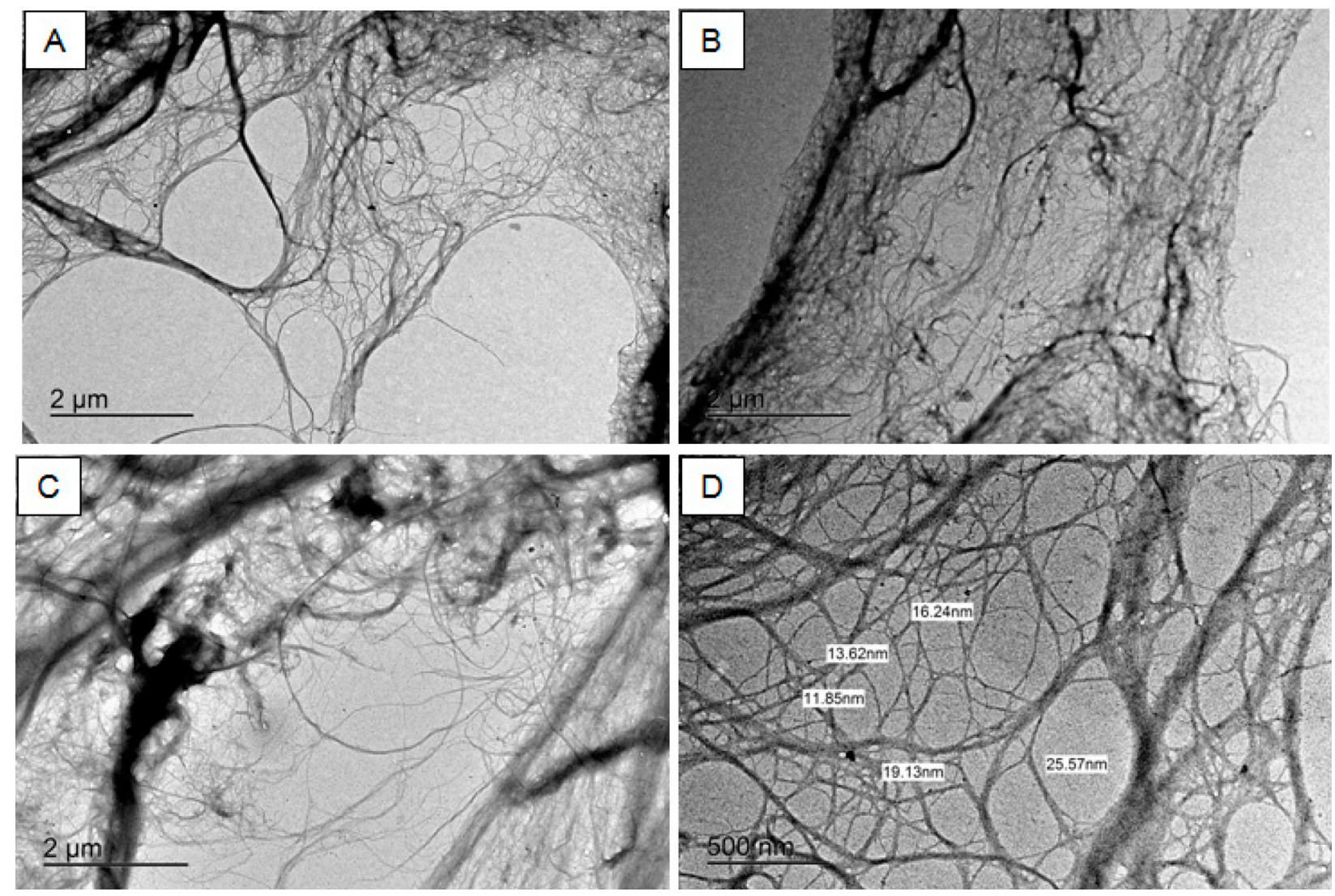

Figura 2. MET de nanofibrilas de celulose. A) nanofibrilas $0,5 \%$ de consistência - 3kx; B) nanofibrilas $1,0 \%$ de consistência - 3kx; C) nanofibrilas $1,5 \%$ de consistência - 3kx; D) diâmetros das nanofibrilas - $10 \mathrm{kx}$.

Figure 2. TEM of celullose nanofibrils. A) nanofibrils $0,5 \%$ wt $-3 \mathrm{kx} ; \mathrm{B}$ ) nanofibrils $1,0 \% \mathrm{wt}-3 \mathrm{kx} ; \mathrm{C}$ ) nanofibrils $1,5 \%$ wt $-3 \mathrm{kx}$; D) diameters of nanofibrils $-10 \mathrm{kx}$.

Considerando nanoestruturas como elementos que apresentam pelo menos uma de suas dimensões menores que 100 nm (STELTE; SANADI, 2009; QUA; HORNSBY, 2011) é possível observar a presença de nanofibrilas de celulose nos materiais obtidos a partir da desfibrilação nas consistências de 0,$5 ; 1,0$ e 1,5\%. De maneira geral, não foi observada diferenças entre as dimensões das nanofibrilas obtidas nos diferentes tratamentos, sendo que o diâmetro médio apresentado foi de 17,28 $(\mathrm{C} 0,5 \%) ; 17,10(\mathrm{C} 1,0 \%)$ e $17,46 \mathrm{~nm}(\mathrm{C} 1,5 \%)$.

A Figura 3 apresenta imagens obtidas por Microscopia de Força Atômica, referentes à celulose nanofibrilada obtida a partir de diferentes consistências.

Para se ter uma ideia da redução do diâmetro das fibras causada pelo processo de desfibrilação no moinho é importante citar o diâmetro dos traqueóides para madeira de Pinus de diferentes espécies encontrado por outros autores. Tomazello Filho (1987) estudando as dimensões dos traqueóides de Pinus oocarpa com 11 anos de idade obteve comprimento médio de 3,9 mm e diâmetro médio de $48 \mu \mathrm{m}$. Os valores encontrados por Nisgoski (2005) para Pinus taeda de diferentes idades também são bem próximos aos anteriores, com largura dos traqueóides de 39,3 e 40,4 $\mu \mathrm{m}$ para árvores de 10 e 17 anos, respectivamente. Em um estudo sobre a variação da dimensão dos traque- 
óides, Irbe et al. (2013) observou valores médios de diâmetros de 36,2 e 35,4 $\mu \mathrm{m}$ para espécies com 27 anos de Pinus sylvestris e Pinus contorta, respectivamente. Dessa forma, a largura das fibras diminuiu consideravelmente de dezenas de micrômetros antes do processo de desfibrilação para dezenas de nanômetros após o processo.
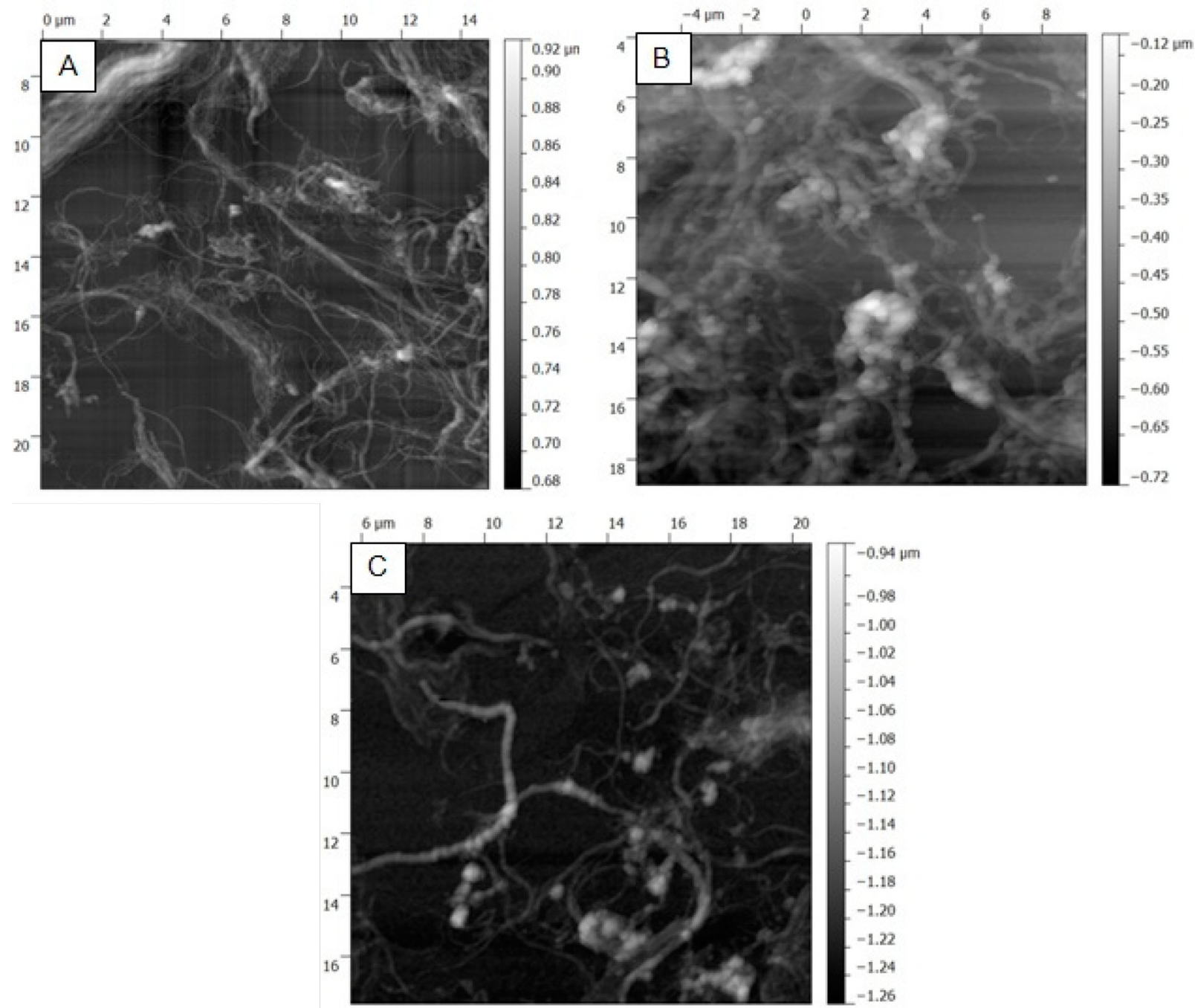

Figura 3. MFA de nanofibrilas de celulose. A) nanofibrilas $0,5 \%$ de consistência; B) nanofibrilas $1,0 \%$ de consistência; C) nanofibrilas $1,5 \%$ de consistência.

Figure 3. AFM of celullose nanofibrils. A) nanofibrils $0,5 \% \mathrm{wt}$; B) nanofibrils $1,0 \% \mathrm{wt}$; C) nanofibrils $1,5 \%$ wt.

\section{Espectroscopia NIR}

Os espectros de absorção na região do NIR dos filmes de celulose nanofibrilada obtidos a partir de diferentes consistências são mostrados na Figura 4. Os espectros foram tratados matematicamente pela normalização.

Na Figura 4 foram indicadas por setas as bandas de absorção características do espectro de materiais lignocelulósicos e os seus respectivos valores em comprimentos de onda $\left(\mathrm{cm}^{-1}\right)$. As bandas características do grupo $\mathrm{OH}$ a $6775 \mathrm{~cm}^{-1}$ podem ser atribuídas à região semi-cristalina da celulose (TSUCHIKAWA; SIESLER, 2003). O pico de $5180 \mathrm{~cm}^{-1}$ deve-se ao OH da água (SIESLER et al., 2002) As bandas entre $4890-4620 \mathrm{~cm}^{-1} \mathrm{e} \mathrm{em} 4280 \mathrm{~cm}^{-1}$ podem ser atribuídas a regiões semi ou cristalinas da celulose (OSBORNE; FEARN, 1986). O pico de $4545 \mathrm{~cm}^{-1}$ corresponde ao estiramento dos grupos $\mathrm{CH}$ aromáticos e a combinação dos grupos $\mathrm{C}=\mathrm{O}$ atribuídos a lignina (WORKMAN; WEYER, 2007).

\section{Índice de Cristalinidade}

As intensidades dos picos cristalinos localizados entre os ângulos de $22^{\circ} \leq 2 \theta \leq 23^{\circ}$ e do halo amorfo $\left(18^{\circ} \leq 2 \theta \leq 19^{\circ}\right)$ para a celulose correspondente aos tratamentos $\mathrm{C} 0,5 \%, \mathrm{C} 1,0 \%$ e $\mathrm{C} 1,5 \%$ estão apresentados na Figura 5. 


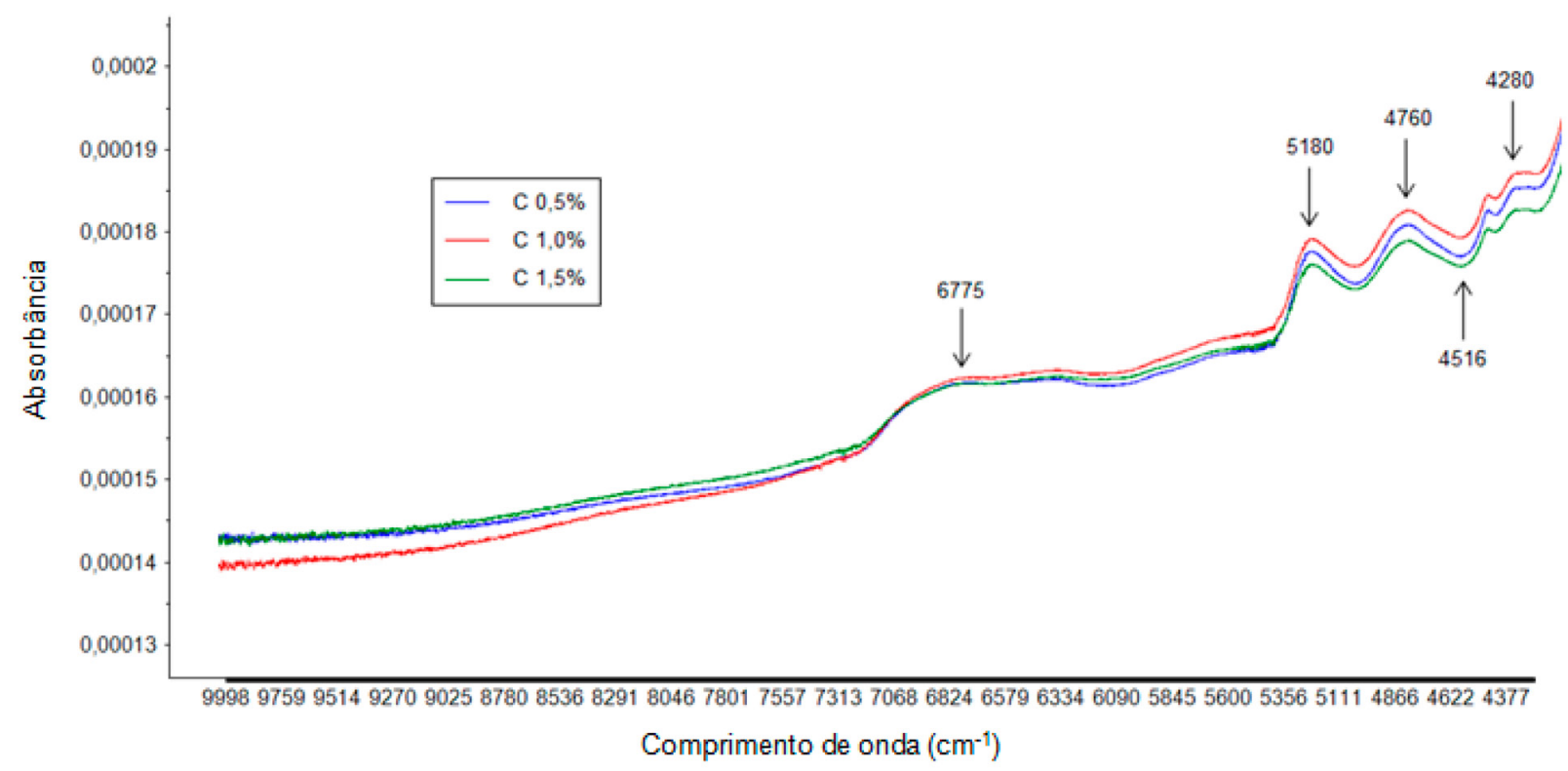

Figura 4. Espectros NIR referentes aos tratamentos $\mathrm{C} 0,5 \%, \mathrm{C} 1,0 \%$ e $\mathrm{C} 1,5 \%$.

Figure 4. NIR spectra refers to $\mathrm{C} 0,5 \%, \mathrm{C} 1,0 \%$ and $\mathrm{C} 1,5 \%$ treatments .

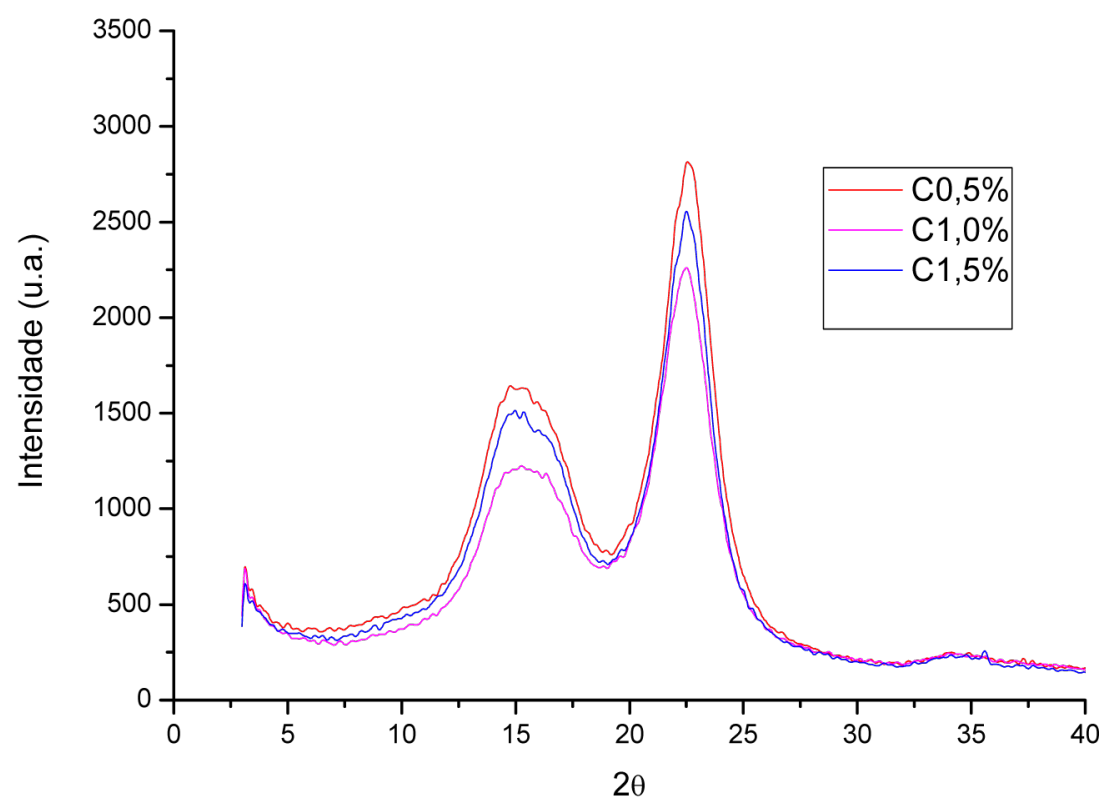

Figura 5. Difratograma de Raios- $X$ da celulose nanofibrilada.

Figure 5. X-ray diffractogram nanofibrillated cellulose.

A determinação do índice de cristalinidade permite analisar a possível degradação sofrida pela celulose na fibra, resultante do processo mecânico de desfibrilação no moinho. O grau de cristalinidade da celulose refere-se à quantidade relativa de celulose na região cristalina onde a fibra apresenta maior resistência à tração e ao alongamento, sendo de grande importância, pois influencia diretamente nas propriedades mecânicas do papel ou filme (FENGEL; WEGENER, 1984).

Os índices de cristalinidade (IC) médios encontrados para cada tratamento estão apresentados na Tabela 2.

Tabela 2. Índice de Cristalinidade médio das nanofibrilas de celulose.

Table 2. Average Crystallinity Index of cellulose nanofibrils.

\begin{tabular}{lc}
\hline Tratamentos & Índice de Cristalinidade médio (\%) \\
\hline C0,5\% & $72,1(\mathrm{a})$ \\
C1,0\% & $69,8(\mathrm{a})$ \\
C1,5\% & $72,2(\mathrm{a})$ \\
CV $(\%)$ & 1,86 \\
\hline
\end{tabular}

Médias seguidas de mesma letra minúscula, na coluna, não diferem entre si pelo teste Tukey, com um nível nominal de significância de 5\%.

Sci. For., Piracicaba, v. 44, n. 110, p. 361-372, jun. 2016

DOI: dx.doi.org/10.18671/scifor.v44n110.09 
Os valores dos índices de cristalinidade obtidos neste estudo foram de 69,8; 72,1 e 72,2\% para as celuloses nanofibrilada obtidas a partir das consistências de 1,0; 0,5 e 1,5\%, respectivamente e não se apresentaram diferentes estatisticamente entre si. Esses valores mostraram-se inferiores quando comparado ao IC da celulose em filmes que não sofreram a desfibrilação (IC= $81,0 \%$ ).

Em pesquisas recentes, autores têm relatado que o tratamento mecânico de desfibrilação no moinho além de provocar a nanofibrilação e exposição das cadeias do polímero de celulose, pode também paralelamente promover a degradação da celulose que pode ser percebida pela redução nos valores do grau de cristalinidade e polimerização, conforme aumenta a intensidade do processo (IWAMOTO et al., 2007; IWAMOTO et al., 2008).

\section{Ensaios Físicos}

A Tabela 3 apresenta os valores médios das propriedades físicas dos nanofilmes de celulose nanofibrilada produzidos e caracterizados nesse estudo.

Tabela 3. Valores médios das propriedades físicas dos filmes.

Table 3. Average values of physical properties of films.

\begin{tabular}{|c|c|c|c|c|c|c|c|c|}
\hline \multirow{2}{*}{ Tratamento } & \multicolumn{2}{|c|}{ Espessura $(\mu \mathrm{m})$} & \multicolumn{2}{|c|}{ Gramatura $\left(\mathrm{g} / \mathrm{m}^{3}\right)$} & \multicolumn{2}{|c|}{ Densidade Ap. $\left(\mathrm{g} / \mathrm{cm}^{3}\right)$} & \multicolumn{2}{|c|}{ Cobb60 $\left(\mathrm{g} / \mathrm{m}^{2}\right)$} \\
\hline & Média & CV & Média & CV & Média & CV & Média & CV \\
\hline $\mathrm{C} 0,5 \%$ & 48 & 4,17 & 58,58 & 7,47 & $1,22(a b)$ & 3,31 & $61,61(a)$ & 3,08 \\
\hline $\mathrm{C} 1,0 \%$ & 47 & 1,24 & 63,08 & 4,24 & $1,35(a)$ & 2,22 & $62,88(a)$ & 3,66 \\
\hline $\mathrm{C} 1,5 \%$ & 53 & 27,21 & 56,92 & 14,34 & $1,10(b)$ & 12,01 & $62,88(a)$ & 3,66 \\
\hline Geral & 49 & 15,93 & 59,53 & 9,33 & 1,22 & 10,98 & 62,46 & 2,90 \\
\hline
\end{tabular}

Médias seguidas de mesma letra minúscula, na coluna, não diferem entre si pelo teste Tukey, com um nível nominal de significância de $5 \%$.

A espessura média apresentada pelos filmes foi de $49 \mu \mathrm{m}$. Os valores de gramatura variaram de 56,92 a $63,08 \mathrm{~g} / \mathrm{m}^{2}$ para os tratamentos $\mathrm{C} 1,5 \%$ e C1,0\%, respectivamente e estão dentro da variação proposta nesse estudo.

A densidade aparente para os filmes pertencentes aos tratamentos $\mathrm{C} 0,5 \%, \mathrm{C} 1,0 \%$ e $\mathrm{C} 1,5 \%$ foi igual a 1,$22 ; 1,35$ e $1,10 \mathrm{~g} / \mathrm{cm}^{3}$, respectivamente. Os valores apresentaram-se diferentes entre si estatisticamente, sendo que os filmes obtidos a partir das nanofibrilas geradas na concentração $1 \%$ destacaram-se pela maior densidade. Os valores médios da densidade obtidos nessa pesquisa foram superiores aos encontrados em outros trabalhos. Stelte e Sanadi, (2009) trabalhando com filmes nanoestruturados relataram valores de densidade aparente de $1 \mathrm{~g} / \mathrm{cm}^{3}$ para filmes obtidos a partir de polpa celulósica de madeiras de fibra longa e Spence et al. (2010) encontraram densidade igual a $0,83 \mathrm{~g} / \mathrm{cm}^{3} \mathrm{em}$ filmes formados por nanofibrilas de polpa branqueada de fibra longa (SPENCE et al., 2010).

Quando comparada a papéis produzidos a partir de polpa celulósica que não sofreu o processo mecânico de desfibrilação, ou seja, que apresenta fibras com diâmetros em torno de $40 \mu \mathrm{m}$, a densidade nos nanofilmes de celulose nanofibrilada mostra-se acentuadamente maior. Klock et al., 2004 avaliando papéis produzidos a partir de polpa Kraft de Pinus taeda com gramatura aproximada de $60 \mathrm{~g} / \mathrm{m}^{2}$, encontraram valores de densidade aparente variando de 0,387 a $0,663 \mathrm{~g} / \mathrm{cm}^{3}$ para os tratamentos sem refino e com 60 minutos de refino a $150 \mathrm{rpm}$, respectivamente.

A maior densidade apresentada pelos filmes formados a partir de nanofibrilas de celulose em relação a papéis de mesma gramatura pode ser explicada pelas menores dimensões apresentadas pela celulose nanofibrilada após as fibras em suspensão sofrerem o processo de desfibrilação no moinho. Durante a obtenção de nanofibrilas de celulose ocorre a redução das dimensões das fibras possibilitando um número maior de ligações e melhor rearranjo das mesmas, levando a formação de uma estrutura mais uniforme e compacta, reduzindo desta forma a espessura dos filmes. Como consequência das reduções na espessura ocorre o aumento das densidades dos filmes nanofibrilados. Celulose nanofibrilada apresenta elevada área específica, cerca de dez vezes maior que a de fibras de celulose, e alta relação entre o comprimento e diâmetro. A maior superfície de contato entre as nanofibrilas proporciona uma maior disponibilidade de grupos $\mathrm{OH}$ e aumento do número de ligações de pontes de hidrogênio formando uma rede mais densa resultando numa maior força e rigidez ao filme (LAVOINE et al., 2012). O aumento na densidade é importante, pois se relaciona fortemente e positivamente com as propriedades mecânicas dos filmes. 
O ensaio de absorção de água pelo método Cobb não mostrou diferença estatisticamente significativa entre os tratamentos avaliados. Os valores foram de $61,61(\mathrm{C} 0,5 \%)$ a $62,88 \mathrm{~g} / \mathrm{m}^{2}(\mathrm{C} 1,0 \% \mathrm{e}$ $\mathrm{C} 1,5 \%)$. Quando comparado a resultados encontrados em outros trabalhos em papéis que não contém a presença de nanofibrilas, filmes nanofibrilados apresentam menor absorção. Andrade (2010) relatou valores de absorção em torno de $140,00 \mathrm{~g} / \mathrm{m}^{2}$ para papéis produzidos com polpa Kraft de Pinus (Kappa 60,0). Viana (2013) encontrou valor médio de absorção Cobb ${ }_{60}$ igual a 195,60 g/m² para papéis produzidos a partir de polpa Kraft de Pinus não branqueada.

A explicação para uma menor absorção de água pode ser também relacionada com o aumento da densidade e por conseqüência a redução da porosidade nos filmes. A estrutura bastante compacta e de menor porosidade que filmes de nanoceluloses, como os de celulose nanofibrilada apresentam, contribuem para a redução na penetração de água (DUFRESNE, 2012). Além da porosidade fatores como a rugosidade da superfície, o teor de umidade e a hidrofobicidade de filmes também exercem influência na resistência dos mesmos à penetração de líquidos (SJÖSTRÖM; ALEN, 1999).

\section{CONCLUSÕES}

Foi possível obter celulose nanofibrilada pelo processo mecânico de desfibrilação a partir das diferentes consistências: $0,5 \%, 1,0 \%$ e 1,5\%. As imagens obtidas dos nanofilmes por microscopia eletrônica de transmissão e microscopia de força atômica indicam que, não foi observada diferenças entre as dimensões das nanofibrilas, que apresentaram diâmetro médio em torno de 17,00 nm.

O processo de desfibrilação mecânica da celulose em diferentes consistência não influenciou nos índices de cristalinidade dos nanofilmes.

Em relação às propriedades físicas dos filmes foi verificada que a presença de nanofibrilas em nanofilmes aumenta a densidade aparente e diminui a absorção de água em filmes nanoestruturados. Para a densidade aparente o tratamento $(\mathrm{C} 1,0 \%)$ destacou-se pelo maior valor apresentado $\left(1,35 \mathrm{~g} / \mathrm{cm}^{3}\right)$. Pelo ensaio de absorção de água pelo método Cobb os nanofilmes não apresentaram diferenças estatísticas significativas com valores de 61,61 (C0,5\%) a 62,88 g/m² (C1,0\%e C1,5\%).

\section{AGRADECIMENTOS}

Os autores agradecem à Coordenação de Aperfeiçoamento de Pessoal de Nível Superior- CAPES e a Fundação Araucária pelo apoio financeiro.

\section{REFERÊNCIAS BIBLIOGRÁFICAS}

$\mathrm{ABE}, \mathrm{K}$; YANO, H. Comparison of the characteristics of cellulose microfibril aggregates isolated from fiber and parenchyma cells of Moso bamboo (Phyllostachys pubescens). Cellulose, v. 17, p. 271-277, 2010.

ANDRADE, A. S. Utilização de micropartículas de lignina kraft combinadas com amido anfótero visando o aumento das propriedades do papel embalagem. 2010, 196p. Tese (Doutorado em Engenharia Florestal) Universidade Federal do Paraná.

ANDRESEN, M.; JOHANSSON, L.S.; TANEM, B.S.; STENIUS, P. Properties and characterization of hydrophobized microfibrillated cellulose. Cellulose, v. 13, p. 665 -677, 2006.

BESBES, I.; VILAR, M.R.; BOUFIA, S. Nanofibrillated cellulose from Alfa, Eucalyptus and Pine fibres: Preparation, characteristics and reinforcing potential. Carbohydrate Polymers, v. 86, p. 1198-1206, 2011.

DUFRESNE, A. Nanocellulose: From Nature to High Performance Tailored Materials. Berlin: Walter De Gruyter Incorporated, 2012. 460p.

FENGEL, D.; WEGENER, G. Wood chemistry, ultrastructure, reactions. New York: Walter de Gruyter, 1984. $612 \mathrm{p}$. 
Potulski et al. - Caracterização de nanofilmes de celulose nanofibrilada obtida em diferentes consistências

FRONE, A.N.; PANAITESCU, D.M.; DONESCU, D. Some aspects concerning the isolation of cellulose microand nano- fibers. UPB Scientific Bulletin, v. 73, p. 133-152, 2011.

GARDNER, D.J.; OPORTO, G.S.; MILLS, R.; SAMIR, M.A.S.A. Adhesion and Surface Issues in Cellulose and Nanocellulose. Journal of Adhesion Science and Technology, v. 22, p. 545-567, 2008.

GONZÁLEZ, I.; BOUFI, S.; PÈLACH, M.A.; ALCALÀ, M.; VILASECA, F.; MUTJÉA, P. Nanofibrillated cellulose as paper additive in Eucalyptus pulps. BioResources, v. 7, nº 4 , p. 5167-5180, 2012.

HUBBE, M.A.; ROJAS, O.J.,LUCIA, L.A.; SAIN, M. Cellulosic nanocomposites: a Review. Bioresources, v. 3, n. 3, p. 929-980, 2008

IOELOVICH, M. Cellulose as a nanostructured polymer: a short review. "Nanostructured cellulose: Review," BioResources, v. 3, n. 4, p. 1403-1418, 2008.

IWAMOTO, S.; KENTARO, A.; YANO, H. The Effect of Hemicelluloses on Wood Pulp Nanofibrillation and Nanofiber Network Characteristics. Biomacromolecules, Uji, v.9, p.1022-1026, 2008.

IWAMOTO, S.; NAKAGAITO, A.N.; YANO, H. Nano-fibrillation of pulp fibers for the processing of transparent nanocomposites. Applied Physics A, v. 89, p. 461-466, 2007.

KLOCK, U.; ANDRADE, A.S.; BITTENCOURT, E.; MOCELIN, E.Z.; CREPALDI, C. Propriedades do papel kraft a partir da madeira juvenil de Pinus maximinoi H.E. Moore e Pinus taeda L. Revista Floresta, v.34, n. 1, 33-44, 2004.

KOLAKOVIC, R.; PELTONEN, L.; LAAKSONEN, T.; PUTKISTO, K.; LAUKKANEN, A.; HIRVONEN, J. SprayDried Cellulose Nanofibers as Novel Tablet Excipient. American Association of Pharmaceutical Scientists, v. 12, n. 4, p. 1366-1373, 2011.

IBER, I.; SABLE, I.; TREIMANIS, A., JANSONS, A.; GRINFELDS, U. Variation in the Tracheid Dimensions of Scots Pine (Pinus sylvestris L.) and Lodgelope Pine (Pinus contorta Dougl. Var. Iatifolia Engelm) Trees Grown in Latvia. Baltic Forestry. v. 19, n.1, p. 120-127, 2013.

LAVOINE, N.; DESLOGES, I.; DUFRESNE, A.; BRAS, J. Microfibrillated cellulose - Its barrier properties and applications in cellulosic materials: A review. Carbohydrate Polymers, v.90, p.735- 764, 2012.

LORANGER, E.; PICHÉ, A.O.; DANEAULT, C. Influence of High Shear Dispersion on the Production of Cellulose Nanofibers by Ultrasound-Assisted TEMPO-Oxidation of Kraft Pulp. Nanomaterials, v.2(3), p. 286$297,2012$.

NAKAGAITO, A.N.; FUJIMURA, A.; SAKAI, T.; HAMA, Y.; YANO, H. Production of microfibrillated cellulose (MFC)-reinforced polylactic acid (PLA) nanocomposites from sheets obtained by a papermaking-like process. Composites Science and Technology, v. 69, p. 1293-1297, 2009.

NISGOSKI, S. Espectroscopia no infravermelho próximo no estudo de características da madeira e papel de Pinus taeda L. 2005. 160 f. Tese (Doutorado em Engenharia Florestal) - Setor de Ciências Agrárias, Universidade Federal do Paraná, Curitiba, 2005.

NOGI, M.; YANO, H. Optically transparent nanofiber sheets by deposition of transparent materials: A concept for a roll-to-roll processing. Applied physics letters, v. 94, p.1-4, 2009.

NOGI, M.; YANO, H. Transparent Nanocomposites Based on Cellulose Produced by Bacteria Offer Potential Innovation in the Electronics Device Industry, Advanced Materials, v. 20, p. 1849-1852, 2008. 
OKAHISA, Y.; YOSHIDA, A.; MIYAGUCHI, S.; YANO, H. Optically transparent wood-cellulose nanocomposite as a base substrate for flexible organic light-emitting diode displays. Composites Science and Technology, v. 69, p. 1958-1961, 2009.

OSBORNE, B.G.; FEARN, T. Near Infrared Spectroscopy in Food Analysis. Longman Scientific and Technical, Harlow, Essex, UK, 1986. 200p.

PANTHAPULAKKAL, S.; SAIN, M. Preparation and Characterization of Cellulose Nanofibril Films from Wood Fibre and Their Thermoplastic Polycarbonate Composites. International Journal of Polymer Scienc, v. 2012, $n^{\circ} .249$, p. 1-6, 2012.

QUA, E.H.; HORNSBY, P.R. Preparation and characterization of nanocellulose reinforcedpolyamide- 6 . Plastics, Rubber and Composites, v.40, nº 6/7, p.300-306, 2011.

SEGAL, L.; CREELY, J.J.; MARTIN, A.E.; CONRAD, C.M. An empirical method for estimating the degree of crystallinity of native cellulose using the X-ray diffractometer. Textile Research Journal, v. 29, n. 10, p. 786794, 1959 .

SEHAQUI, H.; ALLAIS, M.; ZHOU, Q.; BERGLUND, L. A. Wood cellulose biocomposites with fibrous structures at micro- and nanoscale. Composites Science and Technology, n. 71, n. 3, p. 382-387, 2011.

SIESLER, H.W.; OZAKI, Y.; KAWATA, S.; HEISE, H.M. Near infrared spectro scopy: principles, instruments, applications. New York: Wiley-VCH, 2002.

SJÖSTRÖM, E.; ALEN, R. Analytical methods in wood chemistry, pulping, and papermaking. Springer series in wood science. Berlin: Springer, 1999. 316p.

SPENCE, K.; VENDITTI, R.; ROJAS, O.; HABIBI, Y.; PAWLAK, J. The effect of chemical composition on microfibrillar cellulose films from wood pulps: water interactions and physical properties for packaging applications. Cellulose, v. 117, p. 835-848, 2010.

STELTE, W.; SANADI, A.R. Preparation and Characterization of Cellulose Nanofibers from Two Commercial Hardwood and Softwood Pulps. Industrial \& Engineering Chemistry Research, v.48, p.11211-11219, 2009.

STELTE, W.; SANADI, A.R. Preparation and Characterization of Cellulose Nanofibers from Two Commercial Hardwood and Softwood Pulps. Industrial \& Engineering Chemistry Research, v. 48, p.11211-11219, 2009.

SYVERUD, K.; CARRASCO, G.C.; TOLEDO, J.; TOLEDO, P.G. A comparative study of Eucalyptus and Pinus radiata pulp fibres as raw materials for production of cellulose nanofibrils. Carbohydrate Polymers, v. 84 p. 1033-1038, 2011.

TECHNICAL ASSOCIATION OF THE PULP AND PAPER INDUSTRY - TAPPI. T 411-om97. Thickness of paper, paperboard, and combined board. In: Tappi Test Methods. TAPPI Press, Norcross, GA, 2004.

TECHNICAL ASSOCIATION OF THE PULP AND PAPER INDUSTRY - TAPPI. T 410-om02. Grammage of paper and paperboard. In: Tappi Test Methods. TAPPI Press, Norcross, GA, 2004.

TECHNICAL ASSOCIATION OF THE PULP AND PAPER INDUSTRY -TAPPI. T 220-om01. Physical testing of pulp handsheets. In: Tappi Test Methods. TAPPI Press, Norcross, GA, 2004.

TECHNICAL ASSOCIATION OF THE PULP AND PAPER INDUSTRY -TAPPI. T 441-om 98. Physical testing of pulp handsheets. In: Tappi Test Methods. 
TOMAZELLO FILHO, M. Análise da madeira de compressão em Pinus oocarpa estrutura anatômica e quantificação, IPEF, Piracicaba, n. 37, p. 61-68, 1987.

TSUCHIKAWA, S.; SIESLER, H. W. Near-infrared spectroscopy monitoring of the diffusion process of deuterium -labeled molecules in wood. Part II: hardwood. Applied Spectroscopy, v. 57, n. 6, p. 675-681, 2003.

VIANA, L. Desenvolvimento de filmes celulósicos nanoestruturados a partir da polpa Kraft de Pinus sp. 2013.126p. Tese (Doutorado em Engenharia Florestal) - Setor de Ciências Agrárias, Universidade Federal do Paraná, Curitiba, 2013.

WANG, B.; SAIN, M.. Isolation of nanofibers from soybean source and their reinforcing capability on synthetic polymers. Composites Science and Technology. v.67, p.2521-2527, 2007.

WANG, H.; LI, D.; ZHANG, R. Preparation of Ultralong Cellulose Nanofibers and Optically Transparent Nanopapers Derived from Waste Corrugated Paper Pulp. Bioresources, v. 8, n. 1, p. 1374-1384, 2013.

WORKMAN, J.; WEYWER, L. Practical guide to interpretive near-infrared spectroscopy. Boca Raton: CRC, 2007. 332p.

Recebido em 26/03/2015

Aceito para publicação em 11/11/2015 